\title{
Determinação espectrofotométrica do arsênio em solo da cidade de Santana-AP usando o método do dietilditiocarbamato de prata (SDDC) modificado
}

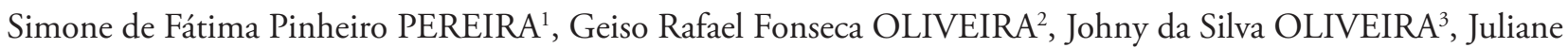
da Silva e SILVA ${ }^{4}$, Pedro Moreira de SOUSA JUNIOR ${ }^{5}$

\section{RESUMO}

Várias toneladas de rejeito de manganês contendo arsênio, gerado por uma empresa de mineraçáo, foram utilizadas como aterro de ruas da cidade de Santana-AP. A possibilidade de exposição das pessoas residentes nessas localidades levou ao estudo de quantificação de arsênio total nos solos. Após a digestão, os teores de arsênio foram quantificados por espectrofotometria de absorção molecular usando um sistema automático de geração de hidretos (HG-MAS), diciclohexilamina/ $\mathrm{CHCl}_{3}$ como solvente do dietilditiocarbamato de prata (SDDC) e $\mathrm{KBH}_{4}$ como redutor. $\mathrm{O}$ método apresentou bons resultados com sensibilidade $(\varepsilon)$ de $1,1010^{4} \mathrm{~L} \cdot \mathrm{mol}^{-1} \cdot \mathrm{cm}^{-1}$, estabilidade de $2,96 \%$ e outras vantagens em relação ao método oficial. O método foi aplicado em amostras de referência de solo com recuperação de 98,82 \% (N=10). As análises de solos mostraram que do total de amostras analisadas $94,74 \%$ apresentaram concentraçáo de arsênio acima do valor editado pela CETESB para solo residencial $\left(50 \mathrm{mg} \cdot \mathrm{kg}^{-1}\right)$ com valor médio de $682,96 \mathrm{mg} . \mathrm{kg}^{-1}$, variando de $48,08 \mathrm{mg} \cdot \mathrm{kg}^{-1}$ a $1.713,00 \mathrm{mg} \cdot \mathrm{kg}^{-1}$ que comprova a contaminação do solo pelo arsênio.

PALAVRAS-CHAVE: Rejeito, contaminação, SDDC

\section{Arsenic spectrophotometric determination in soil of the Santana-AP city using the silver diethyldithiocarbamate (SDDC) modified method}

\section{ABSTRACT}

Several tons of manganese residues containing arsenic, generated by a mining company, were used as streets landfill in SantanaAp city. The possibility of exposure of people living in these villages has led to the study of quantification of total arsenic in the soil. After digestion, the levels of arsenic were quantified by molecular absorption spectrophotometry using a hydrides generation automatic system (HG-MAS), dicyclohexilamine/ $\mathrm{CHCl}_{3}$ as silver diethyldithiocarbamate (SDDC) solvent and $\mathrm{KBH}_{4}$ as reducer. The method presented good results with sensitivity $(\varepsilon)$ of $1.1010^{4} 1 . \mathrm{mol}^{-1} . \mathrm{cm}^{-1}$, stability of $2.96 \%$ and other advantages in relation to the official method. The method was applied to the soil standard samples with recoveries of the $98.82 \%(\mathrm{n}=10)$. The soil analysis showed that $94.74 \%$ samples showed arsenic concentrations above the value published by CETESB for residential land $\left(50 \mathrm{mg} \cdot \mathrm{kg}^{-1}\right)$ with the average value of $682.96 \mathrm{mg} . \mathrm{kg}^{-1}$, ranging of $48.08 \mathrm{mg} \cdot \mathrm{kg}^{-1}$ at $1,713.00$ $\mathrm{mg} \cdot \mathrm{kg}^{-1}$ shows that the soil arsenic contamination.

KEYWORDS: Waste, contamination, SDDC

\footnotetext{
1 Universidade Federal do Pará. Rua Augusto Corrêa S/N - Belém - PA - Brasil, CEP 66075-900. E-mail: simonefp@ufpa.br

2 Universidade Federal do Pará. Rua Augusto Corrêa S/N - Belém - PA - Brasil, CEP 66075-900. E-mail: faeloliveira@oi.com

3 Universidade Federal do Pará. Rua Augusto Corrêa S/N - Belém - PA - Brasil, CEP 66075-900. E-mail: jb_qf@hotmail.com

${ }^{4}$ Universidade Federal do Pará. Rua Augusto Corrêa S/N - Belém - PA - Brasil, CEP 66075-900. E-mail: juliane523@yahoo.com.br

${ }^{5}$ Universidade Federal do Pará. Rua Augusto Corrêa S/N - Belém - PA - Brasil, CEP 66075-900. E-mail: pedromsj@hotmail.com
} 


\section{INTRODUÇÃO}

Uma mineradora lavrou e comercializou minérios de manganês na Serra do Navio, estado do Amapá - Brasil de 1957 até 1997 . Até meados dos anos setenta, os pequenos fragmentos, não encontravam compradores e eram estocados em Serra do Navio. Para a comercialização desses finos foi construída, próxima ao porto, na cidade de Santana-AP, uma usina de pelotização, a qual, usando temperaturas da ordem de 900 a $1000^{\circ} \mathrm{C}$, aglomerava os finos em pelotas endurecidas de cerca de 1 centímetro de diâmetro, permitindo sua venda. As fortes ligaçôes que uniam o arsênio aos hidróxidos foram rompidas devido o minério ter sido aquecido a 1000 graus liberando o mesmo para o meio ambiente. A usina operou de 1973 a 1983, o arsênio é um subproduto da produção de pelotas de manganês quando o minério é submetido a altas temperaturas, após o processamento parte do minério recristalizou em hausmanita e tefroita, minerais instáveis à superfície (Scarpelli, 2002). Em virtude dos processos de pelotização e sinterização originou-se uma grande quantidade de rejeitos. Esses rejeitos foram depositados em uma barragem artificial, situada ao lado da usina de pelotização. Ali, o arsênio solúvel à superfície dos grãos foi dissolvido e contaminou a água da barragem e as águas do subsolo em suas mediaçôes (Scarpelli, 2002). Na época das chuvas a barragem transbordava e acabou por contaminar os rios Elesbão I e II que eram usados pela populaçáo local para diversas finalidades. A secretaria de meio ambiente do Amapá (SEMA-AP) após receber denúncia anônima constatou através de análise da água do rio a contaminação por arsênio, a empresa foi obrigada a retirar o rejeito da barragem e este foi depositado no solo sem proteçáa e coberto por plástico. Este rejeito foi dimensionado ao ser removido da barragem, chegando a 150 mil toneladas, parte dele foi distribuída pela cidade de Santana como aterro de ruas e usado pela populaçấo como carga na construção de casas e como aterro em quintais e jardins. Vários relatos apontam para a ocorrência de problemas de saúde na população que usou o aterro ou mora nas ruas aterradas e também comprovaram a mortandade de animais domésticos e outros problemas.

A presença de arsênio no minério da Serra do Navio já era conhecida pela empresa desde que o mesmo foi caracterizado para comercialização. Os altos níveis de toxicidade de arsênio são bem conhecidos, pois compostos de arsênio, são facilmente absorvidos, tanto oralmente quanto por inalação, sendo a extensão da absorçáo dependente da solubilidade do composto. A toxicidade das várias espécies de arsênio inorgânico varia com a espécie do organismo exposto, com a espécie do arsênio, com a solubilidade da espécie, com a rota de exposição, com a taxa de absorção para o trato gastrointestinal, com a taxa do metabolismo e com a excreção da exposição individual (Sarquis, 1979).
A contaminação por arsênio no ambiente afeta diretamente as populaçóes humanas. Esse elemento, quando presente na cadeia alimentar, irá desencadear uma série de problemas extremamente danosos à saúde levando, inclusive, ao óbito. Sabe-se que o arsênio é carcinógeno para os seres humanos. A inalação de arsênio e, provavelmente, também sua ingestão, resulta em câncer de pulmão. A ingestáo de arsênio causa câncer de pele e de fígado, e, talvez, de bexiga e rins. Existem evidências de que o fumo do cigarro e a exposiçáo simultânea ao arsênio presente no ambiente atuam de maneira sinérgica, causando câncer de pulmão, ou seja, o efeito dos dois fatores juntos é maior que seus efeitos individuais (WHO, 2001).

O efeito letal do arsênio quando consumido em dose aguda é devido a dano gastrointestinal, resultando em diarréia e vômitos intensos. O As (III) inorgânico é mais tóxico que o As $(V)$, embora esse último seja reduzido para o primeiro no corpo humano. Acredita-se que a maior toxicidade do As (III) deve-se à sua capacidade de ser retido no organismo por mais tempo, pois fica ligado a grupos sulfidrila (Valberg et al., 1997).

Devido à importância do arsênio para o meio ambiente, é que foram desenvolvidas técnicas de determinação que permitissem a detecção do arsênio em níveis-traço. Existem muitas técnicas de determinação do arsênio. As mais importantes citadas na literatura são as que usam a técnica da geração de hidreto (arsina) associadas à espectrofotometria de absorçấo atômica (HGAAS) (Mir et al., 2007) e associadas a espectrofotometria de absorçáo molecular como a do SDDC (Arbab-Zavar \& Hashemi, 2000) também conhecida como técnica de Vasak \& Sedivec (1952) metodologia recomendada pela APHA, AWWA e WPCF (1991). O SDDC é dissolvido em piridina e usado como solução absorvedora da arsina, gerada através da reação do zinco em meio clorídrico. A grande desvantagem do método é o desagradável odor da piridina, a pouca estabilidade do complexo formado e aos erros devido à qualidade do zinco usado que dependendo do grau de oxidaçáo e umidade interfere na redução do As (V) a As (III) (Utsumi et al., 1991).

Vários autores pesquisaram a substituição da piridina por outras aminas solubilizadas em clorofórmio (CsikkelSzolnoki et al., 1986; Pande, 1980). O uso de clorofórmio associado a trietanolamina pode servir como solvente para o SDDC em substituição à piridina, faltando esclarecer qual o papel do solvente, quais aminas podem ser utilizadas e porque determinadas aminas possuem desempenho melhor que outras (Pereira et al., 2002). Neste trabalho, a presença de arsênio no solo da cidade de Santana-AP foi avaliada através de uma alternativa ao método do SDDC (Vasak \& Sedivec, 1952) usando a diciclohexilamina em $\mathrm{CHCl}_{3}$ como regente do SDDC e $\mathrm{KBH}_{4}$ como redutor do arsênio. 


\section{MATERIAL E MÉTODOS}

\section{AMOSTRAGEM E TRATAMENTO DA AMOSTRA}

Foram efetuadas coletas de solo da cidade de SantanaAmapá (figura 1), cidade impactada pela contaminaçáo do arsênio com a finalidade de testar o sistema escolhido em amostras naturais.

As amostras foram coletadas na cidade de Santana distante $30 \mathrm{~km}$ de Macapá no estado do Amapá (tabela 1).

Oito ruas em Santana (figura 2) foram inicialmente identificadas como tendo sido aterradas com o rejeito.

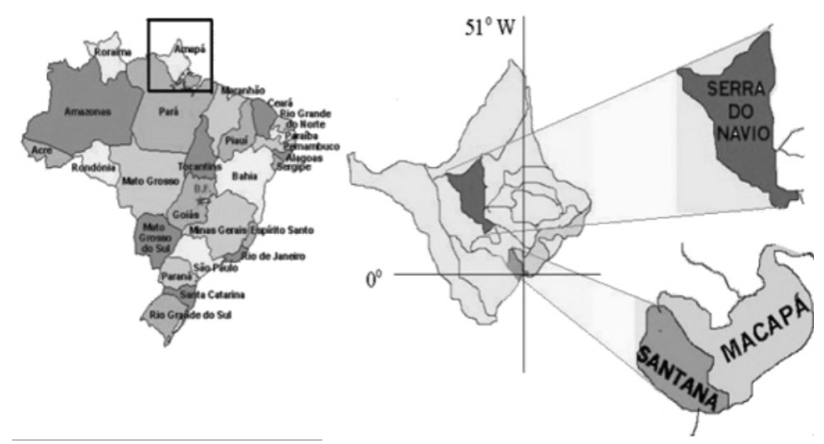

Figura 1 - Mapa de Localização da cidade de Santana - Amapá

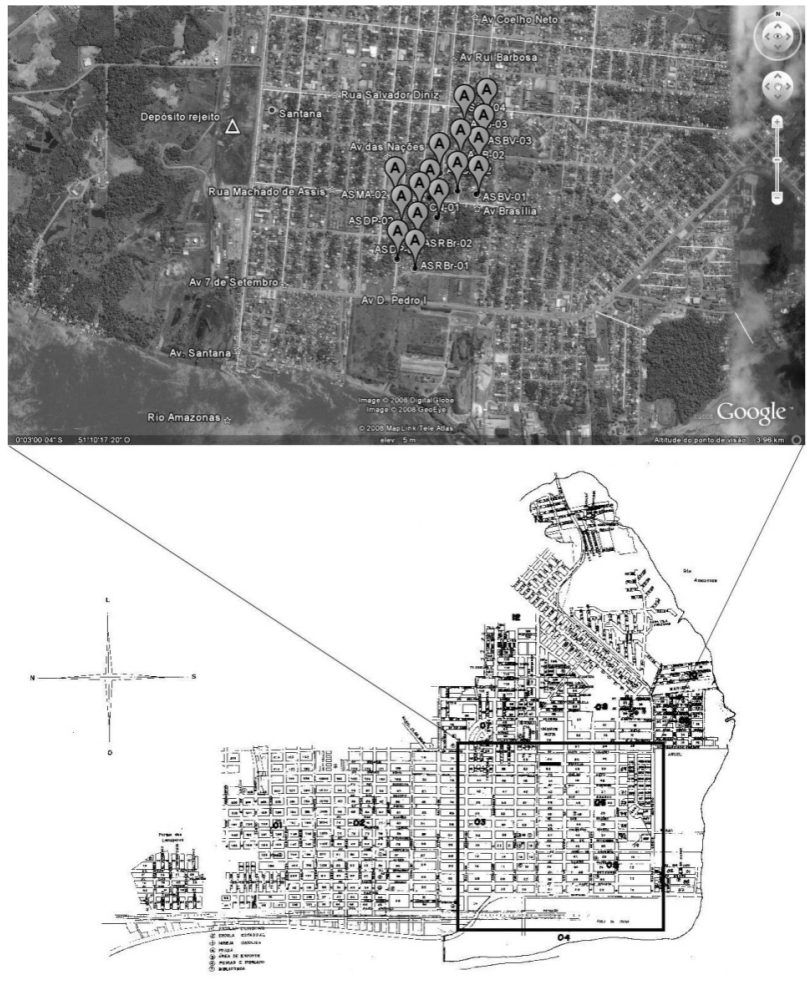

Figura 2 - Mapa de localização da área e pontos de amostragem Fonte: Adaptado do Google Earth, 2008

Tabela 1 - Localização dos pontos de amostragem

\begin{tabular}{|c|c|c|c|c|}
\hline \multirow{2}{*}{$\mathrm{N}^{0}$} & \multirow{2}{*}{ Amostras } & \multicolumn{3}{|c|}{ Localização } \\
\hline & & Local & Latitude & Longitude \\
\hline 01 & ASMA-01 & Rua Machado de Assis & $0^{\circ} 2 ' 55,01 " \mathrm{~S}$ & $51^{\circ} 10^{\prime} 12,97 " 0$ \\
\hline 02 & ASMA-02 & Rua Machado de Assis & $0^{\circ} 2 ' 54,45 " S$ & $51^{\circ} 10^{\prime} 19,62 " 0$ \\
\hline 03 & ASBV-01 & Av. Brasília & $0^{\circ} 2 ' 54,14 " S$ & $51^{\circ} 10^{\prime} 04,31^{\prime \prime} 0$ \\
\hline 04 & ASBV-02 & Av. Brasília & $0^{\circ} 2^{\prime} 48,88^{\prime \prime} S$ & $51^{\circ} 10^{\prime} 03,93^{\prime \prime} 0$ \\
\hline 05 & ASBV-03 & Av. Brasília & $0^{\circ}$ 2'44,22"S & $51^{\circ} 10^{\prime} 03,56^{\prime \prime} 0$ \\
\hline 06 & ASBV-04 & Av. Brasília & $0^{\circ} 2^{\prime} 39,90 " S$ & $51^{\circ} 10^{\prime} 03,23 " 0$ \\
\hline 07 & ASRBr-01 & Av. Rio Branco & $0^{\circ} 3$ '08,02”S & $51^{\circ} 10^{\prime} 15,62 " 0$ \\
\hline 08 & ASRBr-02 & Av. Rio Branco & $0^{\circ} 3$ '02,89"S & $51^{\circ} 10^{\prime} 15,18^{\prime \prime} 0$ \\
\hline 09 & ASRBr-03 & Av. Rio Branco & $0^{\circ} 2 ' 57,58 " S$ & $51^{\circ} 10^{\prime} 14,85^{\prime \prime} 0$ \\
\hline 10 & ASB-01 & Av. Coelho Neto & $0^{\circ} 2$ '53,55"S & $51^{\circ} 10^{\prime} 07,79^{\prime \prime} 0$ \\
\hline 11 & ASB-02 & Av. Coelho Neto & $0^{\circ} 2 ' 47,40 " S$ & $51^{\circ} 10^{\prime} 07,25 " 0$ \\
\hline 12 & ASB-03 & Av. Coelho Neto & $0^{\circ} 2 ' 41,72 " S$ & $51^{\circ} 10^{\prime} 06,83^{\prime \prime} 0$ \\
\hline 13 & ASCN-01 & Av. Rui Barbosa & $0^{\circ} 2 ' 58,49 " S$ & $51^{\circ} 10^{\prime} 11,49^{\prime \prime} 0$ \\
\hline 14 & ASCN-02 & Av. Rui Barbosa & $0^{\circ} 2 ' 50,29 " S$ & $51^{\circ} 10^{\prime} 11,09^{\prime \prime} 0$ \\
\hline 15 & ASDP-01 & Av. Dom Pedro & $0^{\circ} 3$ '06,38"S & $51^{\circ} 10^{\prime} 18,83^{\prime \prime} 0$ \\
\hline 16 & ASDP-02 & Av. Dom Pedro & $0^{\circ} 2 ' 59,77 " S$ & $51^{\circ} 10^{\prime} 18,31^{\prime \prime} 0$ \\
\hline 17 & ASDP-03 & Av. Dom Pedro & $0^{\circ} 2 ' 53,15 " S$ & $51^{\circ} 10^{\prime} 17,62^{\prime \prime} 0$ \\
\hline 18 & ASN-01 & Av. das Nações & $0^{\circ} 3 \prime 03,65 " S$ & $51^{\circ} 10^{\prime} 21,94^{\prime \prime} 0$ \\
\hline 19 & ASN-02 & Av. das Nações & $0^{\circ} 2 ' 58,79 " S$ & $51^{\circ} 10^{\prime} 21,67^{\prime \prime} 0$ \\
\hline
\end{tabular}


As amostras foram coletadas de um perfil de solo de 40 $\mathrm{cm}$. A distância entre os pontos de coleta em uma mesma rua foi de $25 \mathrm{~m}$. As amostras foram coletadas em sacos plásticos etiquetados e conservadas sob refrigeração.

\section{REAGENTES E SOLUÇÕES}

- Solução absorvedora SDDC/DCH em $\mathrm{CHCl}_{3}$ - 0,5\% $(\mathrm{m} / \mathrm{v})$ - dissolver $0,5 \mathrm{~g}$ de SDDC $\left(\mathrm{C}_{5} \mathrm{H}_{10} \mathrm{NAgS}_{2}\right)$ em $100 \mathrm{~mL}$ de diclohexilamina 5\% (v/v) em $\mathrm{CHCl}_{3}$.

- Solução-estoque de arsênio $(1000 \mu \mathrm{g} / \mathrm{mL})$ - dissolver $1,3200 \mathrm{~g}$ de $\mathrm{As}_{2} \mathrm{O}_{3}$ em $10 \mathrm{~mL}$ de água desionizada contendo $4 \mathrm{~g}$ de $\mathrm{NaOH}$. Diluir para $1000 \mathrm{~mL}$ com água desionizada.

- Soluçâao de $\mathrm{KBH}_{4} 10,0 \%$ (m/v) em solução de $\mathrm{NaOH}$ $2,0 \%(\mathrm{~m} / \mathrm{v})$, preparada antes do uso.

\section{GERADOR DE HIDRETO}

O gerador de hidreto utilizado é uma adaptação do gerador recomendado pela APHA, AWWA e WPCF (1991). Este gerador permite a saída da arsina sem perdas significativas, é de fácil manipulação e não possui adaptaçôes, constituindose de um corpo único. A arsina produzida no frasco gerador é borbulhada na solução absorvedora contida no tubo absorvedor, formando o complexo colorido indicativo da presença de arsênio.

A solução redutora é introduzida no frasco gerador através de um tubo capilar usado em sistemas de injeçáo de fluxo (FIA), que é conduzida através do tubo absorvedor até chegar ao frasco gerador usando uma bomba peristáltica de 6 vias. A bomba peristáltica possibilita que a solução de $\mathrm{KBH}_{4}$ possa

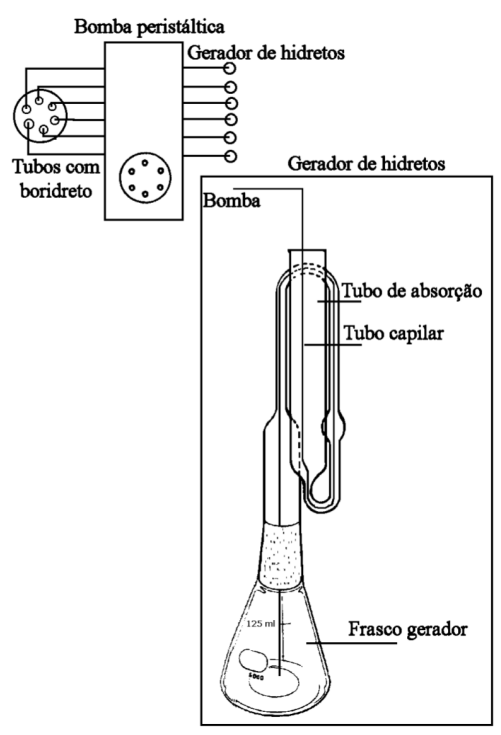

Figura 3 - Sistema gerador de hidreto automático ser introduzida no frasco gerador, com o sistema fechado e fluxo contínuo (figura 3).

Após o uso, os geradores são retirados, lavados com água e extran e secos em estufa a $60^{\circ} \mathrm{C}$.

\section{PROCEDIMENTO}

As amostras foram secas em estufas a $60^{\circ} \mathrm{C}$, trituradas, passadas em peneiras de 20 mesh e quarteadas.

O estudo da digestão foi efetuado em laboratório usando diferentes tipos e quantidades de ácidos, com diferentes potências do microondas. Ao todo foram testados 10 procedimentos com diferentes recuperaçôes do arsênio na amostra certificada, a que apresentou a melhor recuperação foi incluída como a ideal. $\mathrm{O}$ estudo da recuperação é clássico na química analítica e deve ser efetuado em amostra com concentração conhecida, pode ser amostra certificada ou outra amostra qualquer na falta desta, no segundo caso é adicionada uma quantidade conhecida de arsênio em uma das amostras e estas são analisadas com e sem a adição. Depois através de regra de três é efetuado o cálculo da recuperação que será em função da concentração de arsênio no final do processo.

A digestáo que apresentou a melhor recuperaçáo (92,37 \%) foi a que usou $0,01 \mathrm{~g}$ de amostra certificada mais 15 $\mathrm{mL}$ de $\mathrm{HCl}$ concentrado, o aquecimento foi realizado em forno de microonda (marca Provecto) por 7 minutos em potência de $600 \mathrm{w}$ em sistema fechado usando recipiente de hostaflon e bombas com sistema de fechamento tipo camisa em seguida as amostras ainda nas bombas foram resfriadas por 15 minutos em refrigerador a uma temperatura de $4^{\circ} \mathrm{C}$. As bombas foram abertas e $20 \mathrm{~mL}$ de água desionizada foi adicionada. Foi adotada a quantidade de $0,01 \mathrm{~g}$ devido às altas quantidades de As nas amostras e aos erros devido a diluiçôes sucessivas caso fosse usada a quantidade de $0,25 \mathrm{~g}$. Os estudos de repetibilidade com esta quantidade foram satisfatórios apresentando erros abaixo de $5 \%$.

Após solubilização das amostras, foi efetuado o tratamento com carvão ativado e filtragem para remoção de cor e em seguida a amostra certificada foi transferida para balóes de 50 $\mathrm{mL}$ e avolumadas com água desionizada.

No frasco gerador foram adicionados $50 \mathrm{~mL}$ de amostra, $10 \mathrm{~mL}$ de $\mathrm{HCl}$ concentrado (o $\mathrm{pH}$ do meio deve estar entre 1 e 4) e $2 \mathrm{~mL}$ de KI 15,0 \% (m/v) (redutor auxiliar). Em seguida o tubo absorvedor contendo $4 \mathrm{~mL}$ de soluçáo absorvedora e algodão embebido em solução de $\mathrm{Pb}\left(\mathrm{CH}_{3} \mathrm{COO}\right)_{2} 10,0 \%$ $(\mathrm{m} / \mathrm{v})$ na extremidade (é usado para eliminar as interferências de sulfetos) e entáo conectado ao frasco gerador. A arsina gerada pela adiçấo de $6,0 \mathrm{~mL}$ de solução de $\mathrm{KBH}_{4}$ 10,0 \% $(\mathrm{m} / \mathrm{v})$ em NaOH 2,0 \% (m/v) (quantidade de $\mathrm{KBH}_{4}=600$ $\mathrm{mg}$ - vazão da solução de $\mathrm{KBH}_{4}=0,98 \mathrm{~mL} /$ minuto) foi borbulhada na solução absorvedora (6 minutos), um complexo vermelho é entâo formado. As medidas foram tomadas no 
espectrofotômetro UV-Visível, Varian modelo DMS-80, duplo feixe com registrador Intralab utilizando-se células de quartzo de $10 \mathrm{~mm}$ com volume interno reduzido. As análises foram feitas em triplicatas acompanhadas de um branco. O máximo de absorção ocorre em 500 a $510 \mathrm{~nm}$, para a piridina o máximo de absorção ocorreu em $530 \mathrm{~nm}$. Este procedimento foi repetido para as amostras de solo.

\section{RESULTADOS E DISCUSSÃO}

\section{O ESPECTRO DE ABSORÇÃO}

A redução de espécies de arsênio é altamente dependente do $\mathrm{pH}$, entretanto, tanto As (III) como As (V) são reduzidos para arsina em valores de $\mathrm{pH}$ abaixo de 4. Somente As (III) é reduzido em $\mathrm{pH}$ elevado (em torno de 9), acredita-se que todo As (V) é reduzido para As (III) em pH entre 1 e 4. A reação de redução que ocorre na presença de boridreto é mostrada nas equaçóes 1 e 2 .

$\mathrm{As}(\mathrm{V})+2 \mathrm{BH}_{4}^{-}+6 \mathrm{H}_{2} \mathrm{O} \rightarrow \mathrm{As}(\mathrm{III})+2 \mathrm{~B}(\mathrm{OH})_{3}+3 \mathrm{H}_{2}$ (equaçấo 1) seguido da reduçáo do As (III) para arsina,

$\mathrm{As}$ (III) $+3 \mathrm{BH}_{4}^{-}+9 \mathrm{H}_{2} \mathrm{O} \rightarrow \mathrm{AsH}_{3}+3 \mathrm{~B}(\mathrm{OH})_{3}+9 \mathrm{H}_{2} \quad$ (equaçáo 2) em $\mathrm{pH}=5$ a redução do As (V) para As (III) não é favorável, assim pelo controle da acidez da soluçáo redutora é possível inibir a redução do As (V) para As (III) (Pereira, 1997).

Observa-se que o SDDC apresenta caráter pouco iônico e náo requer um solvente muito polar para que ocorra a quebra da ligação do complexo $\mathrm{AsAg}_{3}$.3SDDC liberando a prata coloidal que é, segundo alguns autores, a responsável pela coloração vermelha do complexo (equações 3 e 4).

$\mathrm{AsH}_{3}+6 \mathrm{SDDC} \rightarrow \mathrm{AsAg}_{3} .3 \mathrm{SDDC}+3 \mathrm{HDDC}$ (equação 3)

$\mathrm{AsAg}_{3} \cdot 3 \mathrm{SDDC}+3 \mathrm{NR}_{3}+3 \mathrm{HDDC} \rightarrow 6 \mathrm{Ag}+\mathrm{As}(\mathrm{DDC})_{3}+$ $3\left(\mathrm{NR}_{3} \mathrm{H}\right) \mathrm{DDC}$

(equação 4 )

Bode \& Hachmann (1968) efetuaram um estudo sobre a reação da arsina com o SDDC, soluçóes de piridina, $\mathrm{AsH}_{3} \mathrm{e}$ SDDC em razóes molares variadas foram preparadas, e seus espectros determinados. Estudos com uma ultracentrífuga confirmaram que o complexo vermelho formado pela reação da arsina com o SDDC é devido à natureza coloidal da prata existente no complexo vermelho formado.

O espectro de absorçáo foi construído para 10 e $15 \mu \mathrm{g}$ de arsênio. A maioria dos estudos que usam o método do SDDC usam quantidades ao invés de concentraçôes para o As (Csikkel-Szolnoki et al., 1986; Arbab-Zavar \& Hashemi, 2000), pois estas dependem do volume da soluçáo absorvedora que neste estudo foi de $4 \mathrm{~mL}$. Para que as comparaçôes fossem efetivadas foi adotado o mesmo procedimento. O máximo de absorção encontrado foi em $510 \mathrm{~nm}$ para $4 \mathrm{~mL}$ de solução absorvedora e $50 \mathrm{~mL}$ de solução (figura 4).
Ao diminuir-se a concentração de SDDC de $0,5 \%$ para $0,25 \%$ para as mesmas condiçóes usadas na construçáo do espectro, verificou-se que, ocorre perda na sensibilidade do complexo colorido em estudo. $\mathrm{O}$ sistema diciclohexilamina em $\mathrm{CHCl}_{3}$ apresentou uma melhor sensibilidade $(\varepsilon=9.230,77$ L. $\left.\mathrm{mol}^{-1} \cdot \mathrm{cm}^{-1}\right)$ que o sistema usando piridina $(\varepsilon=8.431,57$ L. $\left.\mathrm{mol}^{-1} \cdot \mathrm{cm}^{-1}\right)$. Um aumento de $799,2 \mathrm{~L} \cdot \mathrm{mol}^{-1} \cdot \mathrm{cm}^{-1}$ significa um aumento em termos de unidades de absorvância comprovando o aumento da sensibilidade do método adotado.

O método recomendado pela APHA, AWWA e WPCF (1991) e que usa a piridina como solvente do SDDC foi testado em paralelo e somente os resultados obtidos foram incluídos neste estudo para efeito de comparaçáo.

No estudo dos interferentes foi observado que o antimônio interfere, pois forma a estibina $\left(\mathrm{SbH}_{3}\right)$ que é destilada juntamente com o arsênio. Os efeitos depressores do antimônio são eliminados pelo uso do EDTA (Pereira, 1997).

\section{EFEITO DA CONCENTRAÇÃO DA AMINA SOBRE 0 ESPECTRO DE ABSORÇÃO}

Para este estudo foram testadas as seguintes concentraçóes da diciclohexilamina (DCH) em $\mathrm{CHCl}_{3}: 0 ; 0,5 ; 1,0 ; 2,0$ ; 5,0 ; 7,0 e $10 \%(\mathrm{v} / \mathrm{v})$. Quando a amina não está presente $\left(0,0 \%\right.$ da amina) o sistema SDDC/ $\mathrm{CHCl}_{3}$ não apresenta a formação de complexo dentro da faixa de comprimento de onda pesquisado.

Ao aumentar-se a concentração da amina, verifica-se que ocorre um incremento na sensibilidade. $\mathrm{O}$ inconveniente está por conta do aumento do sinal do branco, este inconveniente pode ser resolvido pelo uso de carvão ativo e filtragem da solução $\mathrm{DCH} / \mathrm{CHCl}_{3}$.

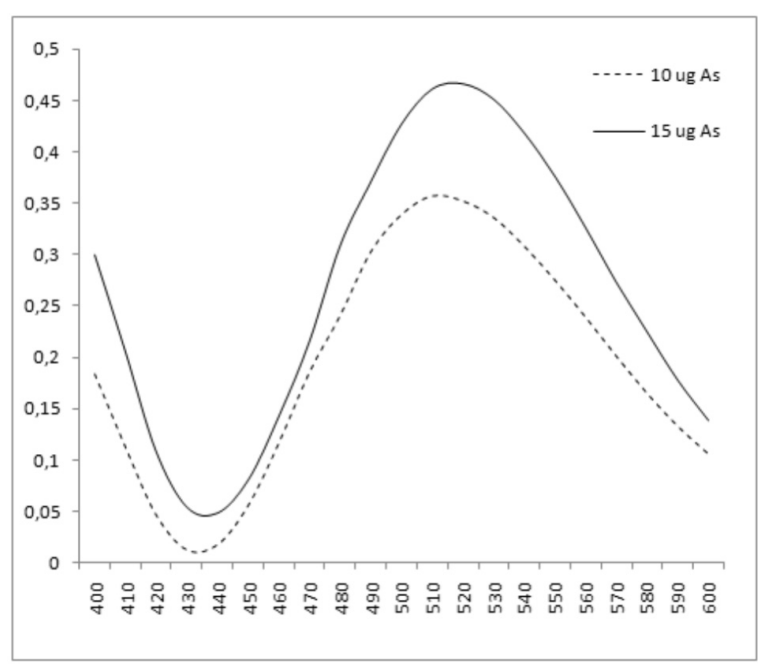

Figura 4 - Espectro de absorção do complexo 


\section{ESTABILIDADE DO COMPLEXO}

Foi efetuada uma comparação entre as estabilidades do complexo usando $5 \%$ e $10 \%$ (v/v) da diciclohexilamina, o tempo de estudo foi de 75 minutos, com intervalos de 5 minutos entre cada leitura. O complexo é estável nas duas concentraçóes. Devido ao aumento do branco na concentração de $10 \%(v / v)$ da amina, náo houve um aumento significativo na sensibilidade do método, assim as duas concentraçôes podem ser usadas, porém recomenda-se o uso dos $5 \%$ (v/v) por apresentar um branco menor. Em seguida, com a solução absorvedora recomendada, foi efetuado o estudo da estabilidade em 240 minutos, o complexo formado se mostrou estável no intervalo de tempo estudado, apresentando uma variação de $2,96 \%$ e desvio padrão de 0,013 . A estabilidade do sistema usando piridina é de apenas 45 minutos.

\section{APLICAÇÃO DA LEI DE BEER}

A curva analítica do complexo SDDC/diciclohexilamina em $\mathrm{CHCl}_{3}$, representada pela equação $\mathrm{y}=0,036 \mathrm{x}-0,007$, demonstrou que o método é linear na faixa de 0 a $25 \mu \mathrm{g}$ de As ou mais, com uma correlação de 0,9995 , com vantagem sobre o método usando a piridina que apresentou desvio da lei de Beer a partir de $20 \mu \mathrm{g}$ de As.

\section{PRECISÃO, LIMITE DE DETECÇÃO, QUANTIFICAÇÃO E EXATIDÃO DO MÉTODO}

$\mathrm{O}$ sistema SDDC/diciclohexilamina em $\mathrm{CHCl}_{3}$ apresentou uma precisão (repetibilidade) de 4,92 \%, com um desvio de $0,026 \%$ para 10 replicatas de uma amostra de solo no qual foi adicionado $15 \mu \mathrm{g}$ de As. Esse resultado é 2,8 vezes superior ao encontrado no método usando SDDC/piridina.

O limite de detecção (LD) (nível de \pm 3s) foi de 5,00 $\mu \mathrm{g}$ de As $\mathrm{kg}^{-1}$, desvio padráo de $0,003 \mu \mathrm{g}$ de $\mathrm{As} \mathrm{kg}^{-1}$, para $50 \mathrm{~mL}$ de solução e 15 replicatas do branco, o resultado é 20 vezes superior ao encontrado para o método usando a piridina. $\mathrm{O}$ limite de quantificaçấo foi de $16,67 \mu \mathrm{g}$ de $A s \mathrm{~kg}^{-1}$, para as mesmas condiçốes do LD. Este resultado possibilita o emprego do método em amostras onde a concentração de arsênio seja a níveis de $\mu \mathrm{g} \cdot \mathrm{kg}^{-1}$, como amostras de água.

No estudo da exatidão do método, foi usada amostra padrão de referência certificada de solo (NIST-2586) que contém as duas espécies de arsênio (As III e As IV) e um conteúdo de arsênio de $8,7 \pm 1,5 \mathrm{mg} \cdot \mathrm{kg}^{-1}$, apresentando uma recuperação analítica (exatidáo) de $98,82 \%(\mathrm{~N}=10)$ e desvio padrão de $0,1324 \mathrm{mg} \cdot \mathrm{kg}^{-1} \mathrm{As}$, o que demonstra a exatidáo do método. O estudo da repetibilidade foi aplicado para amostras de solo certificada apresentando um coeficiente de variação de $5,15 \%$ evidenciando a boa precisão do método.

Também foi feito o estudo da adiçáo e recuperação nas amostras. $\mathrm{O}$ método se mostrou linear quando aplicado para faixas de concentraçóes de arsênio (III) variando de $10 \mathrm{mg} \cdot \mathrm{kg}^{-1}$ a $100 \mathrm{mg} \cdot \mathrm{kg}^{-1}$ de As com uma correlaçáo de 0,9989.

\section{APLICAÇÃO DO MÉTODO}

O método desenvolvido foi aplicado a amostras de solo contaminado com rejeito de uma fábrica de pelotizaçấo. Os resultados encontrados para o solo analisado se encontram na tabela 2. Todos os resultados aqui apresentados são médias de 3 replicatas.

Os resultados mostraram que apenas uma amostra analisada ficou com teor abaixo de $50 \mathrm{mg} \cdot \mathrm{kg}^{-1} \cdot 15,79 \%$ das amostras ficaram com teor de arsênio entre 50 e 100 mg. $\mathrm{kg}^{-1}$ As, $78,95 \%$ estão acima desta faixa e $57,89 \%$ das amostras analisadas apresentaram níveis de arsênio acima de 500 mg.kg ${ }^{-1}$, o maior valor encontrado foi da amostra ASBV-03 com $1.713,00 \pm 591,00 \mathrm{mg} \cdot \mathrm{kg}^{-1} \mathrm{e}$ a de menor valor foi a amostra ASB-03 com 48,08 \pm 16,26 mg.kg-1.

Os resultados mostraram que $94,74 \%$ das amostras estavam com concentração de arsênio acima do limite editado pela CETESB (2000) (50 mg. $\left.\mathrm{kg}^{-1}\right)$ para solos residenciais o que evidencia uma contaminação do solo pelo arsênio. Os valores editados pela CETESB são reconhecidos no Brasil como referência para este tipo de estudo. $\mathrm{Na}$ falta de uma legislaçáo brasileira para solo estes limites são aceitos e usados.

Segundo a WHO (2001) o conteúdo de As na crosta é de 1,5-2 mg.kg-1 ; em solos não contaminados o conteúdo de As pode variar de $0,2 \mathrm{a} 40 \mathrm{mg} \cdot \mathrm{kg}^{-1}$ o que confirma os dados da CETESB como limites de As para solo não contaminado aceitos mundialmente.

Em algumas ruas a presença do rejeito é aparente e em outras uma pequena camada de aterro foi colocada sobre o rejeito visando a minimizar os efeitos danosos causados pela presença do arsênio e de outros metais pesados. A natureza do resíduo descartado é extremamente importante, determinando sua periculosidade em relação ao ambiente e às transformaçóes promovidas no solo. É fundamental que o solo apresente propriedades físicas e químicas que o capacitem a inertizar os resíduos pelo maior tempo possível, evitando liberá-los ao ambiente, principalmente os materiais ricos em metais pesados. Em alguns locais da cidade pesquisada, crianças são expostas ao material, que contamina não só os residentes nas áreas afetadas como também contamina os corpos hídricos locais afluentes do rio Amazonas, as águas subterrâneas, a flora e a fauna dos ecossistemas aquáticos e terrestres.

Este estudo está sendo desenvolvido para uma área de maior abrangência, incluindo locais não afetados e locais de lazer da populaçâo local (campos de futebol, balneários, etc.) onde há informação de deposição de rejeito. Também estão previstos estudos de lixiviação e solubilização e confecção de mapas de risco para subsidiar açôes dos órgãos ambientais locais visando à garantia da saúde da população e ecossistemas. 
Tabela 2 - Resultado da concentração de arsênio em solo

\begin{tabular}{lcc}
\hline $\mathrm{N}^{0}$ & Amostra & $\begin{array}{c}\text { Concentração } \\
\text { de As }\left(\mathrm{mg} \mathrm{kg}^{-1}\right)\end{array}$ \\
\hline 01 & As-01-MA & $1652,26 \pm 96,26$ \\
02 & As-02-MA & $645,02 \pm 29,49$ \\
03 & As-01-BV & $1133,39 \pm 30,40$ \\
04 & As-02-BV & $389,84 \pm 59,15$ \\
05 & As-03-BV & $1713,00 \pm 591,00$ \\
06 & As-04-BV & $1224,81 \pm 137,94$ \\
07 & As-01-RBr & $314,71 \pm 36,45$ \\
08 & As-02-RBr & $581,47 \pm 21,47$ \\
09 & As-03-RBr & $593,00 \pm 77,00$ \\
\hline 10 & As-01-B & $208,83 \pm 83,34$ \\
11 & As-02-B & $89,85 \pm 19,95$ \\
12 & As-03-B & $48,08 \pm 16,26$ \\
13 & As-01-CN & $50,96 \pm 5,29$ \\
\hline 14 & As-02-CN & $100,00 \pm 29,53$ \\
\hline 15 & As-01-DP & $421,25 \pm 67,85$ \\
\hline 16 & As-02-DP & $572,52 \pm 77,97$ \\
\hline 17 & As-03-DP & $869,17 \pm 10,20$ \\
\hline 18 & As-01-N & $1453,33 \pm 207,62$ \\
19 & As-02-N & $914,67 \pm 170,53$ \\
\hline & Média & 682,96 \\
\hline & Desvio padrão & 538,51 \\
\hline & Mínimo & 48,08 \\
\hline & Máximo & $1.713,00$ \\
\hline
\end{tabular}

\section{CONCLUSÕES}

Os resultados mostraram que existe a contaminação do solo nas ruas aterradas com o rejeito de mineração na cidade de Santana. O intemperismo ocasionado pelas chuvas, abundantes na regiáo, pelo vento, etc. e o contato deste material com a população residente poderá gerar a contaminação dos ecossistemas locais (água subterrânea e superficial, biota aquática, plantas, etc.) e a contaminação dos indivíduos. A retirada deste material das ruas aterradas é o primeiro passo para evitar um problema maior, estudos de avaliação da saúde desta população envolvendo toxicologistas e outros profissionais indicarão se a presença do rejeito está ou nấo gerando danos à saúde.

A aplicação da metodologia alternativa de determinação espectrofotométrica do arsênio em solo usando a diciclohexilamina em $\mathrm{CHCl}_{3}$ em substituiçáa a piridina apresentou vantagens em relação ao método tradicional.

O complexo se mostrou estável durante 240 minutos apresentando uma variação de $2,96 \%$ e desvio padráo de 0,013 . A estabilidade do sistema usando piridina é de apenas 45 minutos.
A diciclohexilamina em $\mathrm{CHCl}_{3}$ apresentou uma melhor sensibilidade que o sistema usando piridina.

O método é linear na faixa de 0 a $25 \mu \mathrm{g}$ de As ou mais, com uma correlação de 0,9995 , com vantagem sobre o método usando a piridina que apresentou desvio da lei de Beer a partir de $20 \mu \mathrm{g}$ de As.

Além disso, o método proposto é automatizado diminuindo o tempo de análise que com o zinco é de 45 minutos e com o boridreto de potássio é de apenas 6 minutos.

$\mathrm{O}$ uso do sistema proposto também eliminou o odor desagradável da piridina e reduziu os efeitos danosos causados pelo solvente que é considerado altamente tóxico, além de outras vantagens.

A validade do método alternativo foi comprovada, já que todos os itens exigidos para a validação de metodologia analítica foram seguidos: espectro de absorção, estudo da estabilidade, efeitos variados, linearidade, reprodutibilidade, exatidão, comparação com a metodologia oficial recomendada pela APHA, AWWA e WPCF e aplicação em amostra de solo.

O método pode ser empregado para outras amostras ambientais, como água, sedimento, rocha, etc. onde o arsênio se encontre acima de 5,00 $\mu \mathrm{g} . \mathrm{kg}^{-1}$ de As. O método se apresenta como alternativa rápida e barata em substituição aos métodos instrumentais mais sofisticados como a espectrofotometria de absorção atômica com geração de hidretos (HGAAS) e pode ser adotado em laboratórios com poucos recursos disponíveis.

\section{AGRADECIMENTOS}

Os autores gostariam de agradecer ao CNPq pelo auxílio às bolsas de pesquisa e a Universidade Federal da Bahia aos Profs. Drs. Antonio Celso Spínola Costa e Sérgio Luis Costa Ferreira pelas preciosas orientaçóes do trabalho analítico.

\section{BIBLIOGRAFGIA CITADA}

American Public Health Association (APHA), American Water Works Association (AWWA), Water Pollution Control Federation (WPCF). 1991. Standard methods for the examination of water and wastewater. 13th.Ed., Washington, p. 62-65.

Arbab-Zavar, M.H.; Hashemi, M. 2000. Evaluation of electrochemical hydride generation for spectrophotometric determination of As (III) by silver diethyldithiocarbamate. Talanta, 52(6):10071014.

Bahr, H.; Bahr, H. 1971. Determination of trace amounts of arsenic by the carbamate method. Chemical Analysis, 16(2):427-431.

Bode, H.; Hachmann, K. 1968. Photometrischen arsenbestimmung mit silber diathyldithiocarbamidat. Fresenius'Journal of Analytical Chemistry, 241:18-30. 
Brune, D.; Beltesbrekke, H. 1980. Analysis of arsenic in dental cements according to the silver diethyldithiocarbamate photometric method. Swedish Dental Journal, 4(3):125-128.

CETESB (Companhia de Saneamento do Estado de São Paulo). 2000. Derivação de valores de intervenção para solos e águas subterrâneas, p. 48-73.

Csikkel-Szolnoki, A.; Csakitombacz, E.; Veres, S. 1986. The chromophore serving as basis for the determination of arsenic with silver diethyldithiocarbamate. Acta Chimica Hungarica Models in Chemistry, 123(1-2):45-54.

Google earth. Programa 4.3. Disponível em http://earth.google.com/ intl/pt/. Acessado em 24/03/08.

Gupta, P.K.; Gupta, P.K. 1986. Microdetermination of arsenic in water, spectrophotometrically by arsine silver diethyldithiocarbamatemorpholine-chloroform system. Microchemical Journal, 33:243251.

Han, Y.T.; Yi, C. 1979. Microanalysis of arsenic in seafood and alcoholic beverages. Fen Hsi Hua Hsueh, 7(4):326-327.

Hulanicki, A. 1970. Simultaneous determination of arsenic and antimony by the silver diethyldithiocarbamate method. Chemical Analysis, 15(6):1089-1096.

Lavermann, I. 1976. Determination of arsenic in test materials. Pharmakother Laboratoriumsdiagn, 115(11):1151-1155.

Mir, K.A.; Rutter, A.; Koch, I.; Smith, P.; Reimer, K.J.; Poland, J.S. 2007. Extraction and speciation of arsenic in plants grown on arsenic contaminated soils. Talanta, 72(4):1507-1518.

Onuma, A.; Hamamura, N. 1980. Effect of nitric acid on the determination of arsenic in drinking water. Aichi-Ken Eisei Kenkyusho Ho, 30:27-30.

Pande, S.P. 1980. Morpholine as a substitute for pyridine in determination of arsenic in water. Journal of the Royal Institute of Chemistry, 52(6):256-258.

Pereira, S.F.P. 1997. Alternativas na determinação espectrofotométrica do arsênio através da geração de hidretos. Programa de Pósgraduação em Química. Instituto de Química da UFBa. Tese de doutorado, $166 \mathrm{pp}$.
Pereira, S.F.P.; Ferreira, S.C.; Costa, A.S.; Saraiva, A.F.; Silva, A.F. 2002. Determinação espectrofotométrica do arsênio em cabelo usando o método do dietilditiocarbamato de prata (SDDC) e trietanolamina/ $\mathrm{CHCl}_{3}$ como solvente. Eclética Química, 27:155-170.

Perrin, D.D. 1965. Dissociation constants of organics bases in aqueous solution. London, Butterworths, 146pp.

Sandhu, S.S.; Nelson, P. 1978. Concentration and separation of arsenic from polluted water by ion exchange. Analytical Chemistry, 50(2):322-325.

Sarquis, M. 1979. Arsenic and old myths. Journal of Chemical Education, 56(12):815-818.

Scarpelli, W. 2002. Arsênio do minério de manganês da Serra do Navio-AP. 26pp. (www.cprm.gov.br/pgagem/trabtec.pdf) Acesso: 8/07/2008.

Steckel, L.M.; Hall, J.R. 1962. Spectrophotometric determination of trace arsenic in aluminum alloys, aluminum, uranium, and lead. United States Atomic Energy Commission, Y-1406, 18pp.

Utsumi, S.; Matsuno, S.; Isozaki, A. 1991. Indirect ultraviolet spectrophotometric determination of small amounts of chlorideion with silver-diethyldithiocarbamate complex. Bunseki Kagaku, 40 (6):257-262.

Valberg, P.A.; Beck, B.D.; Bowers, T.S.; Keating, J.L.; Bergstrom, P.D.; Boardman, P.D. 1997. Issues in setting health-based cleanup levels for arsenic in soil. Regulatory Toxicology and Pharmacology, 26: 219-229.

Vasak, V.; Sedivek V. 1952. The colorimetric determination of arsenic, Chemical Listy, 46:341-344.

WHO (World Health Organization). 2001. Arsenic and Arsenic Compounds. Environmental Health Criteria, 224:156-172.

Recebido em 13/08/2008

Aceito em 06/01/2009 